\title{
Estimativas de parâmetros genéticos e de ganhos direto e indireto à seleção para características de crescimento de machos e fêmeas da raça Canchim ${ }^{1}$
}

\author{
Viviane Martha de Castro-Pereira ${ }^{2}$, Maurício Mello de Alencar ${ }^{3^{*}}$, Pedro Franklin Barbosa ${ }^{3}$ \\ 1 Parte da dissertação de Mestrado da primeira autora. Apoio financeiro: CNPq, FAPESP. \\ 2 Pós-graduação da FCAVIUNESP, Departamento de Zootecnia, Jaboticabal, SP. Bolsista da FAPESP. \\ ${ }^{3}$ Embrapa Pecuária Sudeste, Caixa Postal 339, São Carlos, SP, CEP: 13560-970. \\ * Bolsista do CNPq.
}

\begin{abstract}
RESUMO - Neste trabalho, objetivou-se estimar as herdabilidades e as correlações genéticas entre os pesos ao nascimento (PN), à desmama (P240), ao ano (P365) e ao sobreano (P550), os ganhos de peso do nascimento à desmama (GND) e do nascimento ao sobreano (GN18), o número de dias para ganhar $175 \mathrm{~kg}$ do nascimento à desmama (D175) e para ganhar $450 \mathrm{~kg}$ do nascimento ao abate (D450) de machos e fêmeas, o peso adulto (PAD) de fêmeas em um rebanho da raça Canchim visando à definição de critérios de seleção. Foram realizadas análises unicaracteres e bicaracteres pelo método da máxima verossimilhança restrita livre de derivadas utilizando-se modelos estatísticos que incluíram os efeitos fixos de ano e mês de nascimento, sexo do animal, ano e mês do parto, idade do animal ao parto e idade da mãe como covariável (efeitos linear e quadrático), além dos efeitos aleatórios aditivo direto e materno, de ambiente permanente e residual, dependendo da característica. As estimativas de herdabilidade obtidas pelas análises unicaracteres foram iguais a 0,41 (PN), 0,28 (P240), 0,38 (P365), 0,28 (P550), 0,26 (GND), 0,30 (GN18), 0,23 (D175), 0,23 (D450) e 0,48 (PAD), enquanto as estimativas de correlação genética obtidas por análises bicaracteres variaram de 0,28 a 0,97 entre os pesos, de 0,11 a 0,97 entre pesos e ganhos de peso, de 0,00 a -0,98 entre pesos e dias para atingir determinado peso, e de $-0,63$ a $-0,98$ entre os ganhos de peso e dias para atingir determinado peso e iguais a 0,69 para GND e GN18 e 0,76 para D175 e D450. Estas estimativas sugerem a possibilidade de se obter progresso genético pela seleção para todas as características estudadas e que a seleção para qualquer uma delas deverá provocar mudanças nas outras.
\end{abstract}

Palavras-chave: bovinos de corte, correlação genética, herdabilidade, ganho de peso, peso

\section{Estimates of genetic parameters and of direct and correlated responses to selection for growth traits of Canchim cattle}

\begin{abstract}
The objective of this study was to estimate heritability and genetic correlations for body weight at birth (BW), weaning (WW), twelve (W12) and eighteen (W18) months of age, weight gains from birth to weaning (DGBW) and from birth to eighteen months of age (DGB18), days to $175 \mathrm{~kg}$ from birth to weaning (D175 = 175/DGBW), days to $450 \mathrm{~kg}$ from birth to slaughter $(\mathrm{D} 450=450 / \mathrm{DGB} 18)$ and female mature body weight $(\mathrm{ABW})$ using data from a herd of Canchim (5/8 Charolais + 3/8 Zebu) cattle. Univariate and bivariate models including the fixed effects of year and month of birth, sex of calf, year and month of calving, age at calving, age of dam as a covariate (linear and quadratic components) and additive, maternal, permanent environmental and residual random effects were fitted by REML. Heritability estimates obtained by univariate analyses were 0.41 (BW), 0.28 (WW), 0.38 (W12), 0.28 (W18), 0.26 (DGBW), 0.30 (DGB18), 0.23 (D175), 0.23 (D450), and 0.48 (ABW). Genetic correlation estimates ranged from 0.28 to 0.97 between body weight traits, from 0.11 to 0.97 between body weight traits and weight gains, from 0.00 to -0.98 between body weight traits and D175 and D450, and from -0.63 to -0.98 between weight gains and D175 and D450. Genetic correlation estimates were 0.69 between DGBW and DGB18, and 0.76 between D175 and D450. Genetic progress for growth in Canchim cattle is possible by direct as well as by indirect selection on any trait evaluated in this study.
\end{abstract}

Key Words: beef cattle, body weight, genetic correlation, heritability, weight gain

\section{Introdução}

Várias características são consideradas nos programas de avaliação genética de bovinos de corte no Brasil (Alencar, 2002). Entre essas características, destacam-se aquelas relacionadas ao crescimento dos animais que, por apresentarem herdabilidade de magnitude média, serem de fácil medição e estarem diretamente ligadas ao produto final, são mais atraentes aos produtores e, por isso, foram incluídas nesses programas há muitos anos. As características de 
crescimento geralmente utilizadas são os pesos obtidos em determinadas idades (nascimento, desmama, ano e sobreano) e os ganhos de peso em determinados períodos da vida do animal. No Brasil, Silva et al. (2000) e Talhari et al. (2003) verificaram correlação genética positiva entre peso em idades jovens e peso à idade adulta em fêmeas bovinas Canchim, indicando que a seleção para peso deve aumentar o peso à idade adulta das vacas do rebanho. Fries et al. (1996) propuseram as características dias para ganhar $160 \mathrm{~kg}$ entre o nascimento e a desmama e dias para ganhar $240 \mathrm{~kg}$ entre a desmama e o ponto final de abate como critérios de seleção, buscando produzir a mesma unidade em menos tempo.

Para que os programas de melhoramento possam contribuir efetivamente para o aumento da produtividade dos rebanhos de bovinos de corte, é importante avaliar, além das herdabilidades, as relações genéticas e fenotípicas entre características de crescimento que determinam, em parte, a eficiência dos sistemas de produção. Neste trabalho, objetivou-se avaliar, por meio de estimativas de parâmetros genéticos e fenotípicos e de respostas diretas e correlacionadas esperadas, várias características de crescimento, candidatas a critérios de seleção, em um rebanho da raça Canchim.

\section{Material e Métodos}

As informações utilizadas neste trabalho foram coletadas do arquivo de dados do rebanho de bovinos da raça Canchim da Embrapa Pecuária Sudeste, localizada no município de São Carlos, região central do estado de São Paulo.

Foram estudados os pesos ao nascimento (PN), à desmama, aos 12 e aos 18 meses de idade, os ganhos de peso médio diário do nascimento à desmama (GND) e do nascimento ao sobreano (GN18), o número de dias necessários para ganhar $175 \mathrm{~kg}$ do nascimento à desmama (D175) e para ganhar $450 \mathrm{~kg}$ do nascimento ao abate (D450), de machos e fêmeas nascidos de 1953 a 2001 (com exceção de 1970), e o peso adulto (PAD) de fêmeas paridas de 1977 a 2002. Os nascimentos ocorridos no período de 1953 a 1959 foram agrupados em 1959 em virtude do número reduzido de observações em cada ano.

Os animais (machos e fêmeas) do rebanho estudado foram criados em regime exclusivo de pastagens e os cuidados sanitários normais da região foram tomados.

Os pesos à desmama, aos 12 e aos 18 meses de idade foram padronizados para 240 (P240), 365 (P365) e 550 (P550) dias de idade, com base nos ganhos diários do nascimento à desmama, da desmama aos 12 meses e dos 12 aos 18 meses de idade, respectivamente. O número de dias necessários para ganhar $175 \mathrm{~kg}$ do nascimento à desmama (D175) e $450 \mathrm{~kg}$ do nascimento ao abate (D450) foram obtidos dividindo-se 175 e 450 por GND e GN18, respectivamente. Os ganhos de 175 e $450 \mathrm{~kg}$ das características D175 e D450 foram obtidos descontando-se o peso ao nascer dos pesos à desmama e ao abate, considerando-se que um animal Canchim pesa aproximadamente $35 \mathrm{~kg}$ ao nascimento e que $210 \mathrm{~kg}$ seria um bom peso à desmama e, ainda, que $485 \mathrm{~kg}$ seria, segundo Cruz et al. (2004), o peso de abate de animais da raça Canchim.

$\mathrm{O}$ peso à idade adulta das fêmeas (PAD) considerado foi o peso logo após o parto, para vacas de 4, 5, 6 a 8 ou 9 anos de idade. Considerou-se apenas um peso ao parto para cada vaca, aquele aos 6 anos de idade ou o mais próximo dessa idade caso o animal não tivesse o peso aos 6 anos de idade.

A análise de consistência dos dados foi feita utilizando-se procedimentos contidos no SAS (2000). Após essas análises, foram realizadas análises de variância pelo método dos quadrados mínimos, utilizando-se o procedimento GLM (SAS, 2000) para verificar a importância de fontes de variação não-genéticas sobre as características estudadas e considerá-las nas análises para obtenção dos componentes de (co)variância.

As estimativas dos componentes de (co)variância e dos parâmetros genéticos foram obtidas pelo método da máxima verossimilhança restrita livre de derivadas (DFREML) utilizando-se o programa MTDFREML sob modelo animal (Boldman et al., 1993). O modelo estatístico completo utilizado, descrito em termos matriciais, foi:

$$
\mathrm{Y}=\mathrm{Xb}+\mathrm{Zg}+\mathrm{Wm}+\mathrm{Pp}+\mathrm{e}
$$

em que: $\mathrm{Y}=$ vetor de observações; $\mathrm{X}=$ matriz de incidência dos efeitos fixos; $b$ = vetor de efeitos fixos; $Z$ = matriz de incidência dos efeitos genéticos aditivos diretos; $\mathrm{g}=$ vetor de efeitos genéticos aditivos diretos; $\mathrm{W}$ = matriz de incidência dos efeitos genéticos aditivos maternos; $\mathrm{m}=$ vetor de efeitos genéticos aditivos maternos; $\mathrm{P}=$ matriz de incidência dos efeitos de ambiente permanente; $\mathrm{p}$ = vetor de efeitos de ambiente permanente; e e $=$ vetor dos erros associados às observações.

Foram realizadas análises unicaracteres para obtenção dos componentes de variância e estimar a herdabilidade de cada característica e análises bicaracteres para obtenção dos componentes de (co)variância e estimativa das correlações genéticas entre as características estudadas. A matriz de parentesco era composta de 8.865 animais.

Para as características pré-desmame (PN, P240, GND e D175), o modelo estatístico utilizado incluiu os efeitos fixos de sexo, ano e mês de nascimento do animal, além da 
covariável idade da mãe (linear e quadrática), e os efeitos aleatórios aditivos direto e materno, de ambiente permanente e residual. Para as características P365, P550, GN18 e D450, foram considerados apenas os efeitos fixos de sexo, ano e mês de nascimento e os efeitos aleatórios aditivo direto e residual, enquanto, para PAD, consideraram-se os efeitos fixos de ano, mês e idade da vaca ao parto, além dos efeitos aleatórios aditivo direto e residual.

Uma vez obtidas as estimativas de herdabilidade e de correlação genética, as respostas esperadas à seleção direta e indireta e a eficiência relativa da seleção indireta foram calculadas para todas as características estudadas. A resposta direta à seleção foi calculada como $\Delta \mathrm{G}_{\mathrm{X} . \mathrm{x}}=$ $\mathrm{i}_{\mathrm{x}} \cdot \mathrm{h}_{\mathrm{x}}^{2} \cdot \sigma_{\mathrm{PX}}$, em que $\mathrm{i}=$ intensidade de seleção, $\mathrm{h}^{2}=$ herdabilidade da característica sob seleção; e $\sigma_{\mathrm{P}}=$ desvio-padrão fenotípico da característica sob seleção. A resposta correlacionada foi calculada como $\Delta \mathrm{G}_{\mathrm{x} . \mathrm{y}}=$ $\mathrm{i}_{\mathrm{y}} \cdot \mathrm{h}_{\mathrm{x}} \cdot \mathrm{h}_{\mathrm{y}} \cdot \mathrm{r}_{\mathrm{g}} \cdot \sigma_{\mathrm{Px}}$, em que $\mathrm{y}=$ característica sob seleção; $\mathrm{x}=$ característica indiretamente selecionada; e $r_{g}$ = correlação genética entre x e y. A eficiência relativa da seleção indireta (ERS), quanto à resposta direta, foi calculada para cada característica como $\Delta \mathrm{G}_{\mathrm{x} \cdot \mathrm{y}} / \Delta \mathrm{G}_{\mathrm{x} \cdot \mathrm{x}}=\left(\mathrm{r}_{\mathrm{g}} \cdot \mathrm{h}_{\mathrm{y}} \cdot \mathrm{i}_{\mathrm{y}}\right) /\left(\mathrm{h}_{\mathrm{x}} \cdot \mathrm{i}_{\mathrm{x}}\right)$. Para isso, considerou-se retenção de $10 \%(\mathrm{i}=1,75)$ de machos e $50,0 \%(\mathrm{i}=0,80)$ de fêmeas com média de intensidade de seleção de 1,275. Para PAD, não foi calculada a resposta esperada à seleção direta. Nas fórmulas supracitadas, foram utilizadas as estimativas de herdabilidade direta e não as de herdabilidade total descritas por Willham (1972).

\section{Resultados e Discussão}

A estrutura e as estatísticas descritivas das características estudadas podem ser observadas na Tabela 1. Observa-se que as distribuições das características de peso e de ganho de peso se aproximaram da distribuição normal e apresentaram valores de assimetria e de curtose próximos de zero, indicando que as curvas de freqüência desses valores são simétricas e mesocúrticas. Os valores de assimetria e de curtose das características D175 e D450 foram positivos e altos, sugerindo que as curvas de freqüência dos valores dessas características são viesadas para a direita e leptocúrticas. Esse resultado indica que estas duas características merecem algum tipo de tratamento especial ou algum outro tipo de estudo sobre a análise dos dados em uma nova escala ou investigação sobre alguma distribuição teórica diferente da normal. Entretanto, neste trabalho foi utilizada a metodologia de máxima verossimilhança restrita, utilizando-se os dados originais, conforme descrito por outros autores (Marcondes et al., 2000; Ortiz Peña et al., 2004; Garnero et al., 2001; Simonelli et al., 2004).

As estimativas de herdabilidade obtidas neste estudo (Tabela 2) para PN $(0,41)$, P240 $(0,28)$, P365 $(0,38)$ e P550 $(0,28)$ sugerem a possibilidade de ganhos genéticos pela seleção para essas características. As estimativas de PN e P365 estão dentro das amplitudes de 0,34 a 0,76 e de 0,29 a 0,68, enquanto as de P240 e P550 são próximas dos limites inferiores de 0,29 a 0,77 e de 0,41 a 0,54 das apresentadas por outros autores (Alencar et al., 1993; Freitas et al., 1994; Mascioli et al., 1996; Mascioli et al., 1997; Alencar et al.,1998; Silva et al., 2000; Gianlorenço et al., 2003; Mello et al., 2002) para a raça Canchim.

Para o peso à idade adulta, foi obtida estimativa de herdabilidade $(0,48)$ que sugere possibilidades de progresso genético pela seleção. Essa característica é pouco estudada na raça Canchim. Silva et al. (2000) obtiveram valores de 0,38, enquanto Talhari et al. (2003) estimaram valores, para análises bicaracteres com várias características, que variaram de 0,38 a 0,48 (média de 0,42). Rosa et al. (2000, 2001) obtiveram estimativas de herdabilidade inferiores às encontradas neste trabalho para o peso à idade adulta de matrizes Nelore ( 0,36 e 0,26 , respectivamente).

As estimativas de herdabilidade direta para $\operatorname{GND}(0,26)$ e GN18 $(0,30)$ também sugerem a possibilidade de progresso genético pela seleção. Essas características são pouco estudadas na raça Canchim. Oliveira et al. (1982a), Oliveira et al. (1983), Mascioli et al. (1997) e Mascioli et al. (2000) estimaram, respectivamente, herdabilidades de 0,$26 ; 0,38$; 0,37 e 0,39 para GND e Oliveira et al. (1983) obtiveram estimativa de 0,37 para GN18.

Para as características D175 e D450, os valores de herdabilidade foram iguais a 0,23 e indicam que há possibilidade de ganho pela seleção. Na raça Nelore, Marcondes et al. (2000), Ortiz Peña et al. (2004), Garnero et al. (2001) e Simonelli et al. (2004) obtiveram estimativas de herdabilidade para o número de dias para ganhar $160 \mathrm{~kg}$ do nascimento à desmama (D160) que variaram de 0,09 a 0,19 (média de 0,14). Na raça Gir, Muniz et al. (2005) reportaram valor de 0,10 para D160. Para o número de dias para ganhar 240 kg após a desmama na raça Nelore, Ortiz Peña et al. (2000), Garnero et al. (2001) e Simonelli et al. (2004) obtiveram herdabilidades de 0,18 ; 0,16 ; e 0,03 , respectivamente.

As estimativas de herdabilidade materna para as características pré-desmame foram iguais a 0,$05 ; 0,10 ; 0,10$ e 0,08 para PN, P240, GND e D175, respectivamente, e sugerem dificuldades em melhorar a habilidade materna para essas características pela seleção. Na raça Canchim, Alencar et al. (1998) e Mello et al. (2002) estimaram, respectivamente, 
Tabela 1 - Estrutura e estatísticas descritivas dos dados dos pesos ao nascimento (PN) e padronizados para 240 (P240), 365 (P365) e 550 (P550) dias de idade, ganhos em peso do nascimento à desmama (GND) e do nascimento ao sobreano (GN18), números de dias necessários para ganhar $175 \mathrm{~kg}$ do nascimento à desmama (D175) e $450 \mathrm{~kg}$ entre o nascimento e o abate (D450) de machos e fêmeas e peso à idade adulta (PAD) de fêmeas

Table 1 - Structure and descriptive statistics for body weight at birth (BW), weaning (WW; 240 days), 12 (W12; 365 days) and 18 (W18; 550 days) months of age, weight gains from birth to weaning (DGBW) and from birth to 18 (DGB18) months of age, days to $175 \mathrm{~kg}$ from birth to weaning (D175) and days to $450 \mathrm{~kg}(\mathrm{D} 450)$ from birth to slaughter and mature body weight (ABW) of females

\begin{tabular}{|c|c|c|c|c|c|c|c|c|c|}
\hline Item & $\begin{array}{l}\mathrm{PN} \\
B W\end{array}$ & $\begin{array}{c}\text { P } 240 \\
W W\end{array}$ & $\begin{array}{c}\text { P365 } \\
W 12\end{array}$ & $\begin{array}{c}\text { P550 } \\
W 18\end{array}$ & $\begin{array}{c}\text { GND } \\
D G B W\end{array}$ & $\begin{array}{c}\text { GN18 } \\
\text { DGB18 }\end{array}$ & $\begin{array}{c}\mathrm{D} 175 \\
\text { D175 }\end{array}$ & $\begin{array}{c}\mathrm{D} 450 \\
\mathrm{D} 450\end{array}$ & $\begin{array}{l}\text { PAD } \\
A B W\end{array}$ \\
\hline Animais em $\mathrm{A}^{-1}$ (Animals in $A^{-1}$ ) & 8.865 & 8.865 & 8.865 & 8.865 & 8.865 & 8.865 & 8.865 & 8.865 & 8.865 \\
\hline № de registros ( $N$. of records) & 7.152 & 6.126 & 5.448 & 4.585 & 6.127 & 4.616 & 6.127 & 4.615 & 1.100 \\
\hline Anos (Years) & 42 & 41 & 41 & 39 & 41 & 39 & 41 & 39 & 25 \\
\hline Meses (Months) & 11 & 11 & 11 & 11 & 11 & 11 & 11 & 11 & 9 \\
\hline Média $^{1}$ (Mean) & 36 & 204 & 217 & 282 & $701^{3}$ & 449 & 263 & 1049 & 500 \\
\hline Mediana $^{1}$ (Median) & 35 & 204 & 215 & 280 & $702^{3}$ & 444 & 249 & 1012 & 500 \\
\hline Moda $^{1}$ (Mode) & 30 & 190 & 220 & 274 & $667^{3}$ & 500 & 219 & 900 & 440 \\
\hline Mín. ${ }^{1}$ (Min.) & 20 & 90 & 98 & 127 & $209^{3}$ & 167 & 135 & 581 & 305 \\
\hline Máx. ${ }^{1}$ (Max.) & 60 & 351 & 390 & 456 & $1.295^{3}$ & 774 & 839 & 2575 & 734 \\
\hline Desvio-padrão (Standard deviation) & 6 & 37 & 44 & 52 & $151^{3}$ & 92 & 67 & 240 & 69 \\
\hline $\mathrm{CV}, \%$ & 16 & 18 & 20 & 18 & 21 & 21 & 26 & 23 & 14 \\
\hline Assimetria $^{2}$ (Skewness) & 32 & 11 & 35 & 20 & 5 & 20 & 189 & 137 & 20 \\
\hline Curtose $^{2}$ (Kurtosis) & 36 & 16 & 27 & 12 & 14 & 13 & 679 & 365 & -7 \\
\hline
\end{tabular}

${ }^{1} \mathrm{~kg}$, dias e kg/dia para pesos, dias para e ganhos de peso, respectivamente ( $\mathrm{kg}$, days and kg/day for body weight, days to and weight gains, respectively).

$2 \times 100$

$3 \times 1000$.

Tabela 2 - Estimativas dos parâmetros genéticos \pm erros-padrão dos pesos ao nascimento (PN) e aos 240 (P240), 365 (P365) e 550 (P550) dias de idade, ganhos em peso do nascimento à desmama (GND) e do nascimento ao sobreano (GN18), números de dias necessários para ganhar $175 \mathrm{~kg}$ do nascimento à desmama (D175) e $450 \mathrm{~kg}$ entre o nascimento e o abate (D450) e peso adulto (PAD) obtidos por meio de análises unicarácter

Table 2 - Estimates of genetic parameters \pm standard errors for body weight at birth (BW), weaning (WW; 240 days), 12 (W12; 365 days) and 18 (W18; 550 days) months of age, weight gains from birth to weaning (DGBW) and from birth to 18 (DGB18) months of age, days to $175 \mathrm{~kg}$ from birth to weaning (D175) and days to $450 \mathrm{~kg}$ (D450) from birth to slaughter and mature body weight (ABW) obtained by univariate analyses

\begin{tabular}{llll}
\hline Característica & $\mathrm{h}_{\mathrm{a}}{ }^{2}$ & $\mathrm{~h}_{\mathrm{m}}{ }^{2}$ & $\mathrm{c}^{2}$
\end{tabular}

Trait

\begin{tabular}{llccc}
\hline PN $(B W)$ & $0,41 \pm 0,04$ & $0,05 \pm 0,02$ & $0,05 \pm 0,01$ & $-0,07 \pm 0,15$ \\
P240 (WW) & $0,28 \pm 0,04$ & $0,10 \pm 0,03$ & $0,16 \pm 0,02$ & $-0,42 \pm 0,11$ \\
P365 (W12) & $0,38 \pm 0,03$ & - & - & - \\
P550 (W18) & $0,28 \pm 0,03$ & - & $0,16 \pm 0,02$ & - \\
GND (DGBW) & $0,26 \pm 0,04$ & - & - & $-0,44 \pm 0,11$ \\
GN18 (DGB18) & $0,30 \pm 0,03$ & $0,08 \pm 0,03$ & - \\
D175 (D175) & $0,23 \pm 0,04$ & - & - & $-0,40 \pm 0,13$ \\
D450 (D450) & $0,23 \pm 0,03$ & & & - \\
PAD (ABW) & $0,48 \pm 0,07$ & &
\end{tabular}

$\mathrm{h}^{2}{ }^{2}, \mathrm{~h}^{2}{ }_{\mathrm{m}}, \mathrm{c}^{2} \mathrm{er}$ am = herdabilidades direta e materna, efeito de ambiente permanente e correlação genética entre efeito direto e efeito materno, respectivamente $h_{a}^{2}, h^{2}{ }_{m}, c^{2}$ and $r_{a m}=$ direct and maternal heritability, permanent environmental to total phenotypic variance ratio and genetic correlation between direct and maternal effects, respectively.

valores de 0,04 e 0,03 para a herdabilidade materna de PN e de 0,10 e 0,04 para a herdabilidade materna de $\mathrm{P} 240$. Na raça Nelore, Fries et al. (1996), Ortiz Peña et al. (2004) e Simonelli et al. (2004) verificaram os valores de 0,06; 0,10 e 0,06 para a herdabilidade materna de GND, respectivamente. Também na raça Nelore, Fries et al. (1996), Marcondes et al. (2000), Ortiz Peña et al. (2004) e Simonelli et al. (2004) encontraram os valores de 0,12; 0,00; 0,09 e 0,16 para a herdabilidade materna de D160, respectivamente.

As correlações genéticas entre os efeitos aditivo direto e aditivo materno obtidas neste estudo foram de -0,07 (PN),
-0,42 (P240), -0,44 (GND) e -0,40 (D175) e indicam que, para todas essas características, existe antagonismo genético entre os efeitos diretos e os efeitos maternos, sugerindo que a seleção para qualquer uma dessas características com base nos efeitos diretos não será tão efetiva como se espera, visto que a herdabilidade total $\left[\mathrm{h}^{2}{ }_{\mathrm{t}}=\left(\sigma_{\mathrm{a}}{ }^{2}+0,5 \sigma_{\mathrm{m}}{ }^{2}\right.\right.$ $\left.\left.+1,5 \sigma_{\mathrm{am}}\right) / \sigma_{\mathrm{T}}^{2}\right]$ (Willham, 1972) é menor que a direta $\left(\mathrm{h}^{2} \mathrm{a}=\sigma_{\mathrm{a}}{ }^{2} / \sigma_{\mathrm{T}}{ }^{2}\right)$. Na raça Canchim, Alencar et al. (1998) e Mello et al. (2002) obtiveram os valores de -0,06 e -0,04 e de -0,54 e 0,01 para a correlação entre os efeitos direto e materno de PN e de P240, respectivamente. Na raça Nelore, existem 
comunicações na literatura de -0,58 (Eler et al., 1996) para PN e de -0,40 (Eler et al., 1996) e -0,70 (Ribeiro et al., 2001) para peso à desmama. Ainda na raça Nelore, valores de - 0,24 foram reportados para GND (Simonelli et al., 2004) e de -0,77 a 0,02 para D160 (Marcondes et al., 2000; Simonelli et al., 2004).

As estimativas das correlações genéticas entre os efeitos aditivos diretos das características estudadas obtidas pelas análises bicaracteres foram, em geral, muito semelhantes às obtidas pelas análises unicaracteres, com exceção daqueles casos em que as características analisadas apresentaram correlações genéticas próximas de 1,0 (Tabela 3). Neste trabalho, quando uma característica depende da outra, como P240 e GND, D175eGND, P550 eGN18, eD450 eGN18, houvealguma variação nas estimativas de herdabilidade - algumas foram muito diferentes das obtidas pelas análises unicaracteres. Entretanto, as médias das estimativas foram muito próximas das estimativas obtidas pelas análises unicaracteres.

As estimativas de correlação genética direta (Tabela 3) entre os pesos foram todas positivas $(0,28$ a 0,97$)$ e aquelas envolvendo PN foram sempre as mais baixas. Essas estimativas são semelhantes às obtidas por Oliveira et al. (1982b) e Mascioli et al. (1996), que trabalharam com as mesmas características na raça Canchim.
A correlação genética direta entre os ganhos de peso (GND e GN18) foi de 0,69, semelhante aos valores obtidos por Mascioli et al. (2000) para GND e ganho dos 12 aos 18 meses de idade e entre ganho de peso dos 12 aos 18 meses de idade e ganho da desmama aos 12 meses de idade, também na raça Canchim. Oliveira et al. (1983), entretanto, estimaram correlações genéticas altas entre GND e GN18 $(0,90)$ e entre GND e ganho do nascimento aos 12 meses de idade $(0,84)$, também na raça Canchim. Correlação genética de moderada a alta era esperada entre GND e GN18, pois esta última característica engloba a primeira.

A estimativa de correlação genética direta entre as características D175 e D450 foi de 0,76. Entretanto, esperava-se correlação genética de moderada a alta entre essas características, uma vez que a última inclui a primeira.

As estimativas de correlação genética direta entre as características de peso e as de ganho de peso variaram de 0,11 a 0,97 e foram mais baixas quando envolveram PN. Essas estimativas foram semelhantes, em geral, com outras obtidas para a raça Canchim. Assim, Mascioli et al. $(1997 ; 2000)$ relataram correlações genéticas de 0,38 e 0,36 (PN e GND) e de 0,97 e 0,97 (P240 e GND), respectivamente.

Tabela 3 - Estimativas de correlações genéticas (acima da diagonal) e fenotípicas (abaixo da diagonal) e herdabilidades (amplitude e média entre parênteses, na diagonal) dos pesos ao nascimento (PN) e aos 240 (P240), 365 (P365) e 550 (P550) dias de idade, ganhos de peso do nascimento à desmama (GND) e do nascimento ao sobreano (GN18), números de dias necessários para ganhar $175 \mathrm{~kg}$ do nascimento à desmama (D175) e $450 \mathrm{~kg}$ entre o nascimento e o abate (D450) e peso à idade adulta (PAD) obtidos por meio de análises bicarácter

Table 3 - Estimates of genetic (above the diagonal) and phenotypic (below the diagonal) correlations and heritabilities (range and mean within parentheses, diagonal) for body weight at birth (BW), weaning (WW; 240 days), 12 (W12; 365 days) and 18 (W18; 550 days) months of age, weight gain from birth to weaning (DGBW) and from birth to 18 (DGB18) months of age, days to $175 \mathrm{~kg}$ from birth to weaning (D175) and days to $450 \mathrm{~kg}$ (D450) from birth to slaughter and mature body weight ( $A B W)$ obtained by bivariate analyses

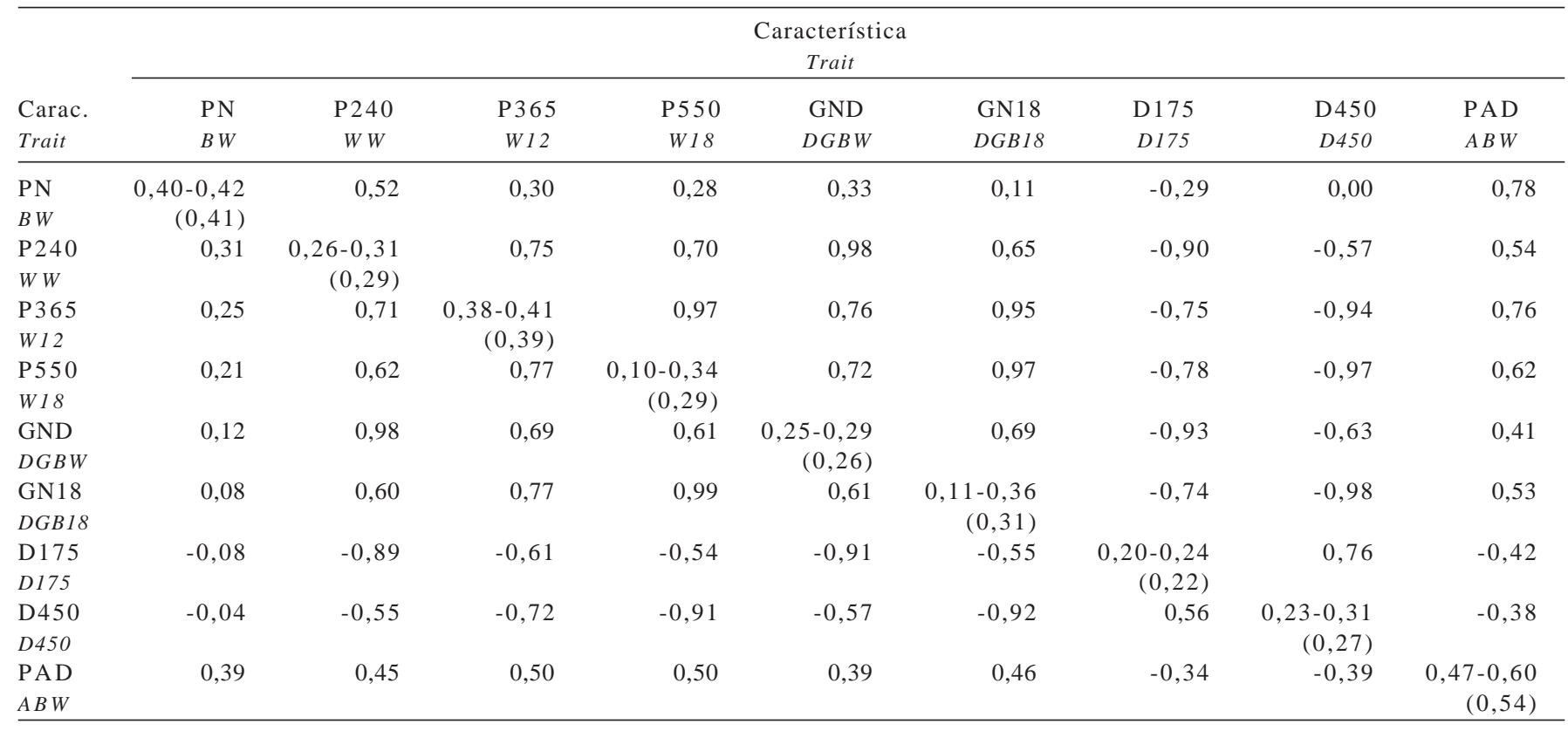


As correlações genéticas diretas entre as características de peso e D175 e D450 foram negativas $(-0,29$ a $-0,98)$, à exceção daquela entre PN e D450, que foi igual a 0,00.

As estimativas das correlações genéticas diretas de GND e GN18 com D175 e D450 foram negativas (-0,63 a -0,98), o que era previsível, pois as características dias para atingir determinado peso são obtidas dividindo-se constantes pelos ganhos de peso. Desse modo, quanto maior o ganho, menor o número de dias para atingir determinado peso. As correlações foram maiores e próximas de $-1,0$ quando os ganhos de peso estiveram diretamente envolvidos no cálculo do número de dias. Os resultados deste trabalho são semelhantes aos descritos por Fries et al. (1996), que estimaram correlação de -0,98 entre os valores genéticos de GND e D160 em animais Nelore. Também são semelhantes aos relatados por Marcondes et al. (2000), que encontraram correlações de -0,86 e -0,80 entre GND e D160 e D300, na raça Nelore. Também na raça Nelore e para GND e D160, Simonelli et al. (2004) relataram correlação genética de -0,95, enquanto Ortiz Peña et al. (2004) estimaram valor de -0,97 para a correlação entre os valores genéticos para as duas características. Ortiz Peña et al. (2000) também estimaram valor de $-0,85$ para o ganho após a desmama e D240. Na raça Gir, Muniz et al. (2005) obtiveram correlação de -0,86 entre os valores genéticos para GND e D160. As estimativas de correlações genéticas entre as características de peso e o peso adulto das fêmeas foram todas positivas e variaram de 0,54 a 0,78 (Tabela 3). Silva et al. (2000) obtiveram o valor de 0,60 para peso à idade adulta de fêmeas e peso de machos aos 365 dias de idade, enquanto Talhari et al. (2003) encontraram correlações de 0,42; 0,66 e 0,60 entre o peso à idade adulta e os pesos à desmama, aos 365 e 550 dias de idade de fêmeas, respectivamente, na raça Canchim. As correlações genéticas de GND $(0,41)$ e GN18 $(0,53)$ com o peso adulto das fêmeas também foram positivas e as estimativas de correlações genéticas entre 0 peso à idade adulta das fêmeas e as características D175 $(-0,42)$ e D450 $(-0,38)$ foram negativas. Esses valores indicam que a seleção para aumento de qualquer um dos pesos e ganhos de peso estudados, desde o nascimento até os 550 dias de idade, ou a redução no número de dias para atingir $175 \mathrm{~kg}$ até a desmama ou $450 \mathrm{~kg}$ até o abate deve resultar em aumento significativo no peso à idade adulta das fêmeas do rebanho.

As estimativas de correlações genéticas entre os efeitos aditivos diretos obtidas neste trabalho, com exceção daquelas envolvendo o peso ao nascimento, indicam que grande parte dos genes de ação aditiva que influenciam qualquer uma das características estudadas também influencia as outras, sugerindo que a seleção para qualquer uma dessas caractéristicas deve resultar em progresso genético nas outras. No caso de seleção para maior peso ou para maior ganho de peso, haverá resposta reduzindo D175 e D450.

As estimativas de correlação genética entre os efeitos maternos obtidas neste estudo foram iguais a 0,14 (PN e GND); 0,27 (PN e P240); 0,98 (P240 e GND); -0,10 (PN e D175); -0,94 (P240 e D175) e-0,94 (GND e D175) e seguiram a mesma tendência das estimativas de correlação genética entre os efeitos aditivos diretos, indicando que, principalmente para as características medidas à desmama, grande parte dos efeitos aditivos maternos que influencia uma característica também influencia a outra. Fries et al. (1996) e Ortiz Peña et al. (2004) estimaram correlações de -0,92 e -0,95 entre os valores genéticos maternos de GND e D160, respectivamente, em animais Nelore. Muniz et al. (2005), na raça Gir, obtiveram correlação de -0,88 entre os valores genéticos maternos para GND e D160. Simonelli et al. (2004), no entanto, estimaram em -0,04 a correlação genética entre os efeitos maternos de GND e D160, também na raça Nelore.

As correlações fenotípicas entre as características estudadas comportaram-se de modo semelhante às correlações genéticas, ou seja, foram, em geral, baixas quando envolveram PN e de médias a altas para as outras características (Tabela 3). Foram também mais altas para características adjacentes e quando uma característica estava envolvida na determinação da outra. Quanto aos sinais, as estimativas foram negativas apenas quando envolveram as características D175 e D450. As estimativas obtidas neste trabalho foram semelhantes às reportadas por Oliveira et al. (1982b), Oliveira et al. (1983), Mascioli et al. (1996), Mascioli et al. (1997) e Mascioli et al. (2000), na raça Canchim, e Marcondes et al. (2000), na raça Nelore.

As respostas esperadas à seleção direta e indireta e a eficiência relativa da seleção indireta (ERS) são apresentadas na Tabela 4. A seleção para qualquer uma das características de crescimento deve provocar mudanças nas outras, mas essas mudanças correlacionadas devem ser, em geral, menores que as mudanças diretas. A seleção indireta só é mais eficiente que a direta nos seguintes casos: 1) seleção para P240 e resposta em GND (ERS = 1,02); 2) seleção para P550 e resposta em D450 (ERS = 1,07); 3) seleção para GN18 e resposta em D450 (ERS = 1,12); e 4) seleção para P365 e resposta em P550 (ERS = 1,13), GN18 (ERS = 1,07) e D450 (ERS = 1,21). Mascioli et al. (1996), usando a mesma metodologia adotada neste trabalho, também para a raça Canchim, verificaram que a seleção para P365 e P550 é mais eficiente para mudar o peso aos 24 meses de idade que a seleção direta para esta característica. 
Tabela 4 - Respostas diretas (diagonal em negrito) e correlacionadas (fora da diagonal) por geração e eficiências relativas da seleção indireta (valores entre parênteses)

Table 4 - Direct (diagonal, bold face) and correlated (off diagonal) selection responses per generation and relative efficiency of indirect selection (within parenthesis) on growth traits

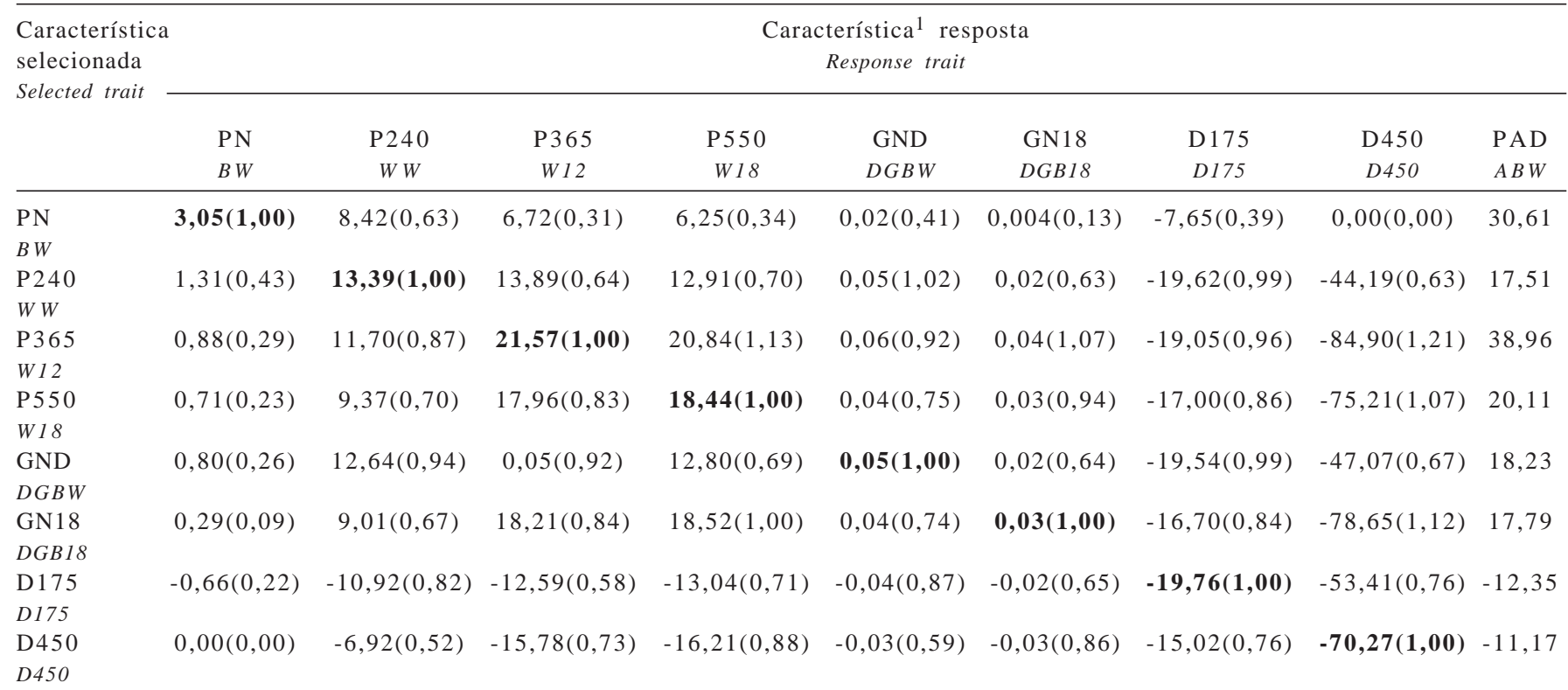

1 PN, P240, P365, P550, GND, GN18, D175, D450 e PAD = pesos (kg/geração) ao nascimento, aos 240, 365 e 550 dias de idade, ganhos de peso (kg/dia/ geração) do nascimento à desmama e do nascimento ao sobreano, números de dias (dias/geração) necessários para ganhar 175 kg do nascimento à desmama e $450 \mathrm{~kg}$ do nascimento ao abate e peso à idade adulta ( $\mathrm{kg} / \mathrm{geração)} \mathrm{das} \mathrm{vacas,} \mathrm{respectivamente.}$

${ }^{1} \mathrm{BW}, W W, W 12, W 18, D G B W, D G B 18, D 175, D 450$ and $A B W=$ body weight (kg/generation) at birth, weaning, 12 and 18 months of age, weight gain ( $\left.k g / d a y / g e n e r a t i o n\right)$ from birth to weaning and from birth to 18 months of age, days (days/generation) to $175 \mathrm{~kg}$ from birth to weaning and days to $450 \mathrm{~kg}$ from birth to slaughter, and mature body weight (kg/generation), respectively.

É importante verificar que a seleção para qualquer uma das características de crescimento medidas após a desmama não resulta em grandes mudanças no peso ao nascimento. Mascioli et al. (1996) obtiveram índices ERS iguais a 0,580; 0,439 e 0,177 quando a seleção foi feita para P240, P365 e P550, em relação à seleção direta para PN. A seleção para todas as características medidas do nascimento aos 18 meses de idade provoca aumentos no peso adulto das vacas.

\section{Conclusões}

As estimativas de herdabilidade obtidas sugerem a possibilidade de se obter progresso genético pela seleção para as características estudadas.

As correlações genéticas obtidas, em geral, indicam que a seleção para aumentar qualquer das características deve resultar em aumento nas outras. A seleção para qualquer das características de crescimento do nascimento aos 18 meses de idade deve ser acompanhada de monitoramento do peso à idade adulta das vacas para evitar aumentos no tamanho das vacas do rebanho.

\section{Literatura Citada}

ALENCAR, M.M. Critérios de seleção em bovinos de corte no Brasil. In: SIMPÓSIO NACIONAL DE MELHORAMENTO ANIMAL, 4., 2002, Campo Grande. Anais... Campo Grande: Sociedade Brasileira de Melhoramento Animal, 2002. (CD-ROM).

ALENCAR, M.M.; BARBOSA, P.F.; BARBOSA, R.T. Parâmetros genéticos para peso e circunferência escrotal em touros da raça Canchim. Revista da Sociedade Brasileira de Zootecnia, v.22, n.4, p.572-583, 1993.

ALENCAR, M.M.; TREMATORE, R.L.; BARBOSA, P.F. et al. Efeitos da linhagem citoplasmática sobre características de crescimento em bovinos da raça Canchim. Revista Brasileira de Zootecnia, v.27, n.2, p.272-276, 1998.

BOLDMAN, K.; KRIESE, L.; Van VLECK, L.D. A manual for use of MTDFREML: a set of programs to obtain estimates of variances and covariances (DRAFT). Lincoln: Department of Agriculture, Agriculture Research Service, 1993. 120p.

CRUZ, G.M.; TULLIO, R.R.; ESTEVES, S.N. et al. Peso de abate de machos não-castrados para a produção do bovino jovem. 2 . Peso, idade e características de carcaça. Revista Brasileira de Zootecnia, v.33, n.3, p.646-657, 2004.

ELER, J.P.; FERRAZ, J.B.; SILVA, P.R. Parâmetros genéticos para peso, avaliação visual e circunferência escrotal na raça Nelore, estimados por modelo animal. Arquivo Brasileiro de Medicina Veterinária e Zootecnia, v.48, n.2, p.203-213, 1996.

FREITAS, A.R.; FAVORETTI, A.C.; ALENCAR, M.M. et al. Uso da máxima verossimilhança restrita e transformação canônica 
para estimação de parâmetros genéticos de características de crescimento em bovinos. Revista da Sociedade Brasileira de Zootecnia, v.23, n.3, p.394-401, 1994.

FRIES, L.A.; BRITO, F.V.; ALBUQUERQUE, L.G. Possíveis conseqüências de seleção para incrementar pesos às idadespadrão vs. reduzir idades para produzir unidades de mercado. In: REUNIÃO ANUAL DA SOCIEDADE BRASILEIRA DE ZOOTECNiA, 33., 1996, Fortaleza. Anais... Fortaleza: Sociedade Brasileira de Zootecnia, 1996. v.1, p.310-312.

GARNERO, A.V.; LÔBO, R.B.; BEZERRA, L.A.F. et al. Comparação entre alguns critérios de seleção para crescimento na raça Nelore. Revista Brasileira de Zootecnia, v.30, n.3, p.714-718, 2001.

GIANLORENÇO, V.K.; ALENCAR, M.M.; TORAL, F.L.B. et al. Herdabilidades e correlações genéticas de características de machos e fêmeas, em um rebanho da raça Canchim. Revista Brasileira de Zootecnia, v.32, n.6, p.1587-1593, 2003 (supl.1).

MARCONDES, C.R.; BERGMANN, J.A.G.; ELER, J.P. et al. Análise de alguns critérios de seleção para características de crescimento na raça Nelore. Arquivo Brasileiro de Medicina Veterinária e Zootecnia, v.52, n.1, p.83-89, 2000.

MASCIOLI, A.S.; ALENCAR, M.M.; BARBOSA, P.F. et al. Estimativas de parâmetros genéticos e proposição de critérios de seleção para pesos na raça Canchim. Revista da Sociedade Brasileira de Zootecnia, v.25, n.1, p.72-81, 1996.

MASCIOLI, A.S.; FARO, L.; ALENCAR, M.M. et al. Estimativas de parâmetros genéticos e fenotípicos e análise de componentes principais para características de crescimento na raça Canchim. Revista Brasileira de Zootecnia, v.29, n.6, p.1654-1660, 2000.

MASCIOLI, A.S.; PAZ, C.C. P.; FARO, L. et al. Estimativas de parâmetros genéticos e fenotípicos para características de crescimento até a desmama em bovinos da raça Canchim. Revista Brasileira de Zootecnia, v.26, n.4, p.709-713, 1997.

MELLO, S.P.; ALENCAR, M.M.; SILVA, L.O.C. et al. Estimativas de (Co)variâncias e tendências genéticas para pesos em um rebanho Canchim. Revista Brasileira de Zootecnia, v.31, n.4, p.1707-1714, 2002.

MUNIZ, C.A.S.D.; CARVALHEIRO, R.; FRIES, L.A. et al. Dois critérios de seleção na pré-desmama em bovinos da raça Gir. 2. Efeito na classificação dos animais. Revista Brasileira de Zootecnia, v.34, n.3, p.816-826, 2005.

OLIVEIRA, J.A.; DUARTE, F.A.M.; LÔBO, R.B. et al. Genetic and phenotypic parameters of birth weight and weaning weight in Canchim cattle. Revista Brasileira de Genética, v.5, n.1, p.131-145, 1982a.

OLIVEIRA, J.A.; DUARTE, F.A.M.; LÔBO, R.B. et al. Genetic analysis of performance traits in Canchim cattle. I. Body weight. Revista Brasileira de Genética, v.5, n.4, p.687-698, 1982 b.
OLIVEIRA, J.A.; DUARTE, F.A.M.; LÔBO, R.B. et al. Genetic analysis of performance traits in Canchim cattle. II. Weight gain. Revista Brasileira de Genética, v.6, n.1, p.81-92, 1983.

ORTIZ PEÑA, C.D.; QUEIROZ, S.A.; FRIES, L.A. Análise comparativa de critérios de seleção para precocidade de crescimento pós-desmama em bovinos Nelore no Paraguai. In: REUNIÃO ANUAL DA SOCIEDADE BRASILEIRA DE ZOOTECniA, 37., 2000, Viçosa, MG. Anais... Viçosa, MG: Sociedade Brasileira de Zootecnia, 2000. p.191.

ORTIZ PEÑA, C.D.; CARVALHEIRO, R.; QUEIROZ, S.A. et al. Comparison of selection criteria for pre-weaning growth traits of Nelore cattle. Livestock Production Science, v.86, p.163167, 2004.

RIBEIRO, M.N.; PIMENTA FILHO, E.C.; MARTINS, G.A. et al. Herdabilidade para efeitos direto e materno de características de crescimento de bovinos Nelore no estado da Paraíba. Revista Brasileira de Zootecnia, v.30, n.4, p.1124-1227, 2001.

ROSA, A.N.; LÔBO, R.B.; OLIVEIRA, H.N. et al. Variabilidade genética do peso adulto de matrizes em um rebanho Nelore do estado de São Paulo. Revista Brasileira de Zootecnia, v.29, n.6, p.1706-1711, 2000.

ROSA, A.N.; LÔBO, R.B.; OLIVEIRA, H.N. et al. Peso adulto de matrizes em rebanhos de seleção da raça Nelore no Brasil. Revista Brasileira de Zootecnia, v.30, n.3, p.1027-1036, 2001 (supl. 1).

STATISTICAL ANALYSIS SYSTEM - SAS. SAS/STAT: user's guide. Version 8.12. Cary: 2000. (CD-ROM).

SILVA, A.M.; ALENCAR, M.M.; FREITAS, A.R. et al. Herdabilidade e correlações genéticas para peso e perímetro escrotal de machos e características reprodutivas e de crescimento de fêmeas, na raça Canchim. Revista Brasileira de Zootecnia, v.29, n.6, p.2223-2230, 2000 (supl. 2).

SIMONELLI, S.M.; SILVA, M.A.; SILVA, L.O.C. et al. Critérios de seleção para características de crescimento em bovinos da raça Nelore. Arquivo Brasileiro de Medicina Veterinária e Zootecnia, v.56, n.3, p.374-384, 2004.

TALHARI, F.M.; ALENCAR, M.M.; MASCIOLI, A.S. et al. Correlações genéticas entre características produtivas de fêmeas em um rebanho da raça Canchim. Revista Brasileira de Zootecnia, v.32, n.4, p.880-886, 2003.

WILLHAM, R.L. The role of maternal effects in animal breeding: III - Biometrical aspects of maternal effects in animals. Journal of Animal Science, v.35, p.1288-1302, 1972. 